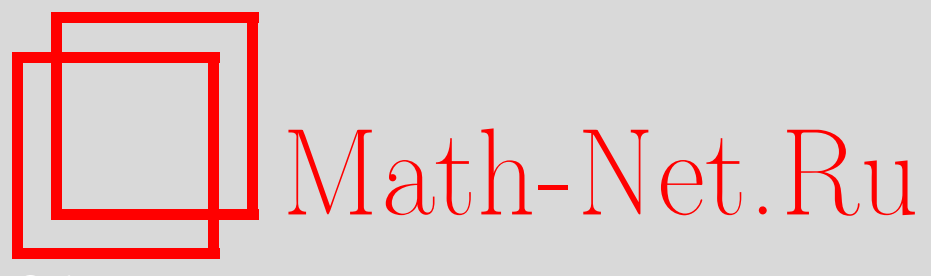

Д. А. Ложников, С. А. Сергеев, О поведении локализованного решения волнового уравнения в окрестности точки локализации при малых временах, Матем. заметки, 2012, том 91, выпуск 1, 149-153

DOI: https://doi.org/10.4213/mzm9297

Использование Общероссийского математического портала Math-Net.Ru подразумевает, что вы прочитали и согласны с пользовательским соглашением http://www.mathnet.ru/rus/agreement

Параметры загрузки:

IP : 3.82 .47 .9

26 апреля 2023 г., $07: 29: 50$

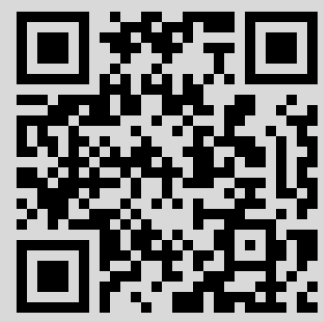




\section{О поведении локализованного решения волнового уравнения в окрестности точки локализации при малых временах}

\section{Д. А. Ложников, С. А. Сергеев}

1. Введение. В работах [1], [2] было построено асимптотическое решение задачи Коши

$$
\eta_{t t}-\nabla C^{2}(x) \nabla \eta=0,\left.\quad \eta\right|_{t=0}=V\left(\frac{x}{\mu}\right),\left.\quad \eta^{\prime}\right|_{t=0}=0, \quad x \in \mathbb{R}^{2},
$$

где $C(x)>0$ гладкая функция, а функция $V(y)$ убывает на бесконечности быстрее, чем $1 /|y|^{\delta}, \delta>1$.

При $t \geqslant t_{0}$, где $t_{0}$ положительно и не зависит от $\mu$, поведение решения достаточно хорошо изучено: при $t=0$ решение локализованно в окрестности точки $x=0$, а при $t>t_{0}$ оно локализовано в окрестности замкнутой кривой, возможно негладкой и с точками самопересечения. При этом поведение решения по времени от 0 до $t_{0}$ изучено значительно хуже. В то же время этот вопрос представляет интерес с точки зрения анализа волны (например, волны цунами) в момент ее возникновения [3]. Цель этой работы изучить поведение решения на указанном промежутке вермени $\left(0, t_{0}\right)$ при предположении, что $t_{0} \sim$ $\mu$. Рассматриваемая задача интересна в теории длинных волн в жидкости над неровным дном, задаваемым уравнением $C^{2}(x)=H(x)$, где $H(x)$ - глубина бассейна.

2. Определение асимптотического решения. Согласно работам [1], [2] асимптотическое решение задачи (1) строится следующим образом. Введем в фазовом пространстве $\mathbb{R}_{p, x}^{4}$ лагранжево многообразие

$$
\Lambda_{2}^{0}=\{p=\mathbf{n}(\psi), x=\alpha \cdot \mathbf{n}(\psi), \mathbf{n}(\psi)=(\cos (\psi), \sin (\psi))\} .
$$

Рассмотрим гамильтонову систему

$$
\dot{x}=\frac{p}{|p|} C(x), \quad \dot{p}=-|p| \nabla C, \quad H(x, p)=|p| C(x) .
$$

Эта система определяет каноническое преобразование (фазовый поток $g_{H}^{t}$ ), применение которого к многообразию $\Lambda_{2}^{0}$ дает семейства, зависящих от $t$ лагранжевых многообразий

$$
\Lambda_{2}^{t}=g^{t} \Lambda_{2}^{0}
$$

Пусть $K_{\Lambda_{2}^{t}}^{h}$ - канонический оператор Маслова на многообразии $\Lambda_{t}^{2}$. Тогда решение задачи (1) представимо в виде

$$
\eta(x, t)=\operatorname{Re}\left\{\sqrt{\frac{\mu}{2 \pi \cdot i}} \cdot \int_{0}^{\infty} \sqrt{\rho} \cdot K_{\Lambda_{2}^{t}}^{\mu / \rho} \widetilde{V}(\rho \mathbf{n}(\psi)) d \rho\right\} .
$$

Здесь $\widetilde{V}(p)$ - проеобразование Фурье от функции $V(y)$.

Задача состоит в упрощении формулы (4) при $t<T \cdot \mu$, где $T>0$ - константа. Для этого упрощения достаточно найти многообразие $\Lambda_{t}^{2}$ приближенно.

Теорема. Главный член в асимптотике решения задачи (1) имеет вид

$$
\eta(x, t)=\frac{1}{2 \sqrt{2 \pi}} \cdot \operatorname{Re}\left\{\int_{0}^{2 \pi} \int_{0}^{\infty}\left[\rho \cdot \widetilde{V}(\rho \mathbf{n}(\psi)) \cdot e^{i \rho / \mu \cdot(\langle\mathbf{n}(\psi), x\rangle-C(0) \cdot t-\langle\nabla C(0), x\rangle \cdot t)}\right] d \psi d \rho\right\} .
$$

Работа была выполнена при поддержке Российского фонда фундаментальных исследований (грант № 08-01-00726) и Министерства образования и науки РФ (грант № 2.1.1/4540). 
Лемма. Для многообразия $\Lambda_{2}^{t}$ справедливы приближенные формулы при малых време$\operatorname{Hax}$

$$
\left(\begin{array}{c}
\mathscr{X} \\
\mathscr{P}
\end{array}\right)=\left(\begin{array}{c}
\alpha \cdot \mathbf{n}(\psi) \\
\mathbf{n}(\psi)
\end{array}\right)+t \cdot\left(\begin{array}{c}
C(\alpha \cdot \mathbf{n}(\psi)) \cdot \mathbf{n}(\psi) \\
-\nabla C(\alpha \mathbf{n}(\psi))
\end{array}\right)+o(t) .
$$

ДокАзАтельство. Доказательство этого факта легко следует из разложения решения системы (2) в окрестности точки $t=0$ по формуле Тейлора с точностью $o(t)$.

Многообразие $\Lambda_{2}^{t}$ можно покрыть такими же картами, как и при $t=0$ (см. [4]). Точнее, имеем четыре карты $\Omega_{i}, i=1,2,3,4$, которые являются гомеоморфными в полосе и представляют собой декартово произведение прямой на дугу окружности. Карте с номером $i$ соответствует дуга окружности

$$
-\frac{\pi}{4}+\frac{(i-1) \pi}{2}-\delta<\psi<\frac{\pi}{4}+\frac{(i-1) \pi}{2}+\delta,
$$

где $\delta$ - некоторое малое положительное число.

В картах 1, 3 фаза $\Phi$ имеет вид $\Phi=\alpha-\mathscr{P}_{2} \cdot \mathscr{X}_{2}+p_{2} \cdot x_{2}$, а в картах $2,4-\Phi=$ $\alpha-\mathscr{P}_{1} \cdot \mathscr{X}_{1}+p_{1} \cdot x_{1}$. Разлагая фазу в ряд Тейлора с точностью до членов первого порядка по переменным $\alpha$ и $\psi$ в окрестности точки $\left(\psi=\psi^{0}, \alpha=0\right)$, получаем, что в каждой карте фаза представима в виде

$$
\Phi=\left\langle P\left(\psi^{0}, t\right), x-X\left(\psi^{0}, t\right)\right\rangle .
$$

ЗАмечание. Формулы (5) описывают асимптотическое решение в случае, когда дно представляет собой наклонную плоскость, описываемую уравнением $C(x)=\nabla C(0) x$.

ДокАЗАТЕЛЬСтво теоремы. В работе [2] было сформулировано утверждение о том, что асимптотическое решение задачи $(1)$ в особых картах с координатами $\left(p_{1}, x_{2}\right)$ определяется следующей формулой:

$$
\begin{aligned}
\eta(x, t)=\operatorname{Re}\left\{\int_{0}^{\infty}\right. & \int_{-\infty}^{\infty}\left[\frac{\rho\left|P_{1 \psi}\right|}{\sqrt{\left|\mathscr{P}_{1 \alpha} \mathscr{X}_{2 \psi}-\mathscr{P}_{1 \psi} \mathscr{X}_{2 \alpha}\right|}} \cdot \tilde{V}(\rho \mathbf{n}(\psi)) \cdot \mathbf{e}(\psi) e^{i \rho / \mu(\langle P(\psi, t), x-X(\psi, t)\rangle)}\right] d \psi d \rho \\
& \left.\times e^{-(i \pi / 2) \cdot \operatorname{Ind}(r *)} \cdot \frac{1}{2 \sqrt{2 \pi}}+O(\mu)\right\} .
\end{aligned}
$$

Учтем в этом выражении разложение (6). Тогда с точностью до $O(t)$ имеем

$$
\mathscr{P}_{1 \alpha} \mathscr{X}_{2 \psi}-\mathscr{P}_{1 \psi} \mathscr{X}_{2 \alpha}=\sin ^{2} \psi, \quad P_{1 \psi}=\sin \psi .
$$

Аналогичное представление решение $\eta(x, t)$ будет иметь и в картах 1,3 . В них якобиан будет иметь вид

$$
\mathscr{X}_{1 \alpha} \mathscr{P}_{2 \psi}-\mathscr{X}_{1 \psi} \mathscr{P}_{2 \alpha}=\cos ^{2} \psi
$$

а в числителе будет стоять $\left|P_{2 \psi}\right|=|\cos \psi|$. Далее при вычислении фазы (7) отбросим слагаемые порядка $O(t)$. В итоге получим

$$
\langle P(\psi, t), x-X(\psi, t)\rangle=\langle\mathbf{n}(\psi), x\rangle-C(0) \cdot t-\langle\nabla C(0), x\rangle \cdot t .
$$

Поскольку решение рассматривается при малых временах, когда на фронте отсутствуют фокальные точки, за исключением фокальной точки в момент времени $t=0$, то величина $\operatorname{Ind}\left(r^{*}\right)=0$. После этого, действуя также, как и в работе [4], с учетом того, что сумма по картам пропадает, так как фаза в каждой карте определяется одной и той же формулой, а корни из якобианов сокращаются с соответствующими производными $\left|P_{k \psi}\right|, k=1,2$, получаем формулу (5). 
3. Решение для источника специального вида. Дальнейшие упрощения формулы (5) связаны с выбором источника специальноговида [5], [6]:

$$
\begin{gathered}
\eta^{0}=V\left(\frac{x}{\mu}\right), \quad V(y)=V_{0}(T(\theta) y), \\
V_{0}(z)=\frac{1}{\left(1+\left(z_{1} / b_{1}\right)^{2}+\left(z_{2} / b_{2}\right)^{2}\right)^{3 / 2}}, \quad T(\theta)=\left(\begin{array}{cc}
\cos \theta & \sin \theta \\
-\sin \theta & \cos \theta
\end{array}\right) .
\end{gathered}
$$

Здесь $b_{1}, b_{2}, \theta$ - действительные параметры. Преобразованием Фурье функции $V(y)$ является функция $\widetilde{V}\left(p_{1}, p_{2}\right)=e^{-|p| \cdot \beta(\psi-\theta)}$, поэтому

$$
\widetilde{V}(\rho \mathbf{n}(\psi))=e^{-\rho \cdot \beta(\psi-\theta)},
$$

где

$$
\beta(\phi)=\sqrt{b_{1}^{2} \cdot \cos ^{2} \phi+b_{2}^{2} \cdot \sin ^{2} \phi} .
$$

УтвеРЖДЕниЕ. Для источника (8) справедлива формула

$$
\begin{aligned}
\eta(x, t)= & \frac{1}{2 \sqrt{2 \pi}} \cdot \operatorname{Re}\left\{\int_{0}^{2 \pi} \frac{d \psi}{\left(\beta(\psi)-i / \mu \cdot\left(\left\langle\mathbf{n}^{0}(\psi), x\right\rangle-C(0) \cdot t-\langle\nabla C(0), x\rangle \cdot t\right)\right)^{2}}\right\} \\
& +O(\mu)+O(t) .
\end{aligned}
$$

ДокАЗАТЕЛЬСтво. Подставляем источник вида (8) в интеграл (5) и, интегрируя по частям, получаем, что

$$
\int_{0}^{\infty} \rho \cdot e^{\rho(-\beta+i / \mu \Phi)} d \rho=\frac{1}{(\beta-i / \mu \cdot \Phi)^{2}},
$$

где $\Phi=\langle\mathbf{n}(\psi), x\rangle-C(0) \cdot t-\langle\nabla C(0), x\rangle \cdot t$. Отсюда следует формула (9).

Случай симметричного источника. В случае, когда источник является симметричным, т.е. $b_{1}=b_{2}=b$, указанный интеграл можно вычислить явно. В этом случае формула для возвышения свободной поверхности жидкости будет иметь вид

$$
\eta(x, t)=\frac{1}{2 \sqrt{2 \pi}} \cdot \operatorname{Re}\left\{\int_{0}^{2 \pi} \frac{d \theta}{(b+i t / \mu \cdot(C(0)+\langle\nabla C(0), x\rangle)-i|x| / \mu \cdot \cos \theta)^{2}}\right\},
$$

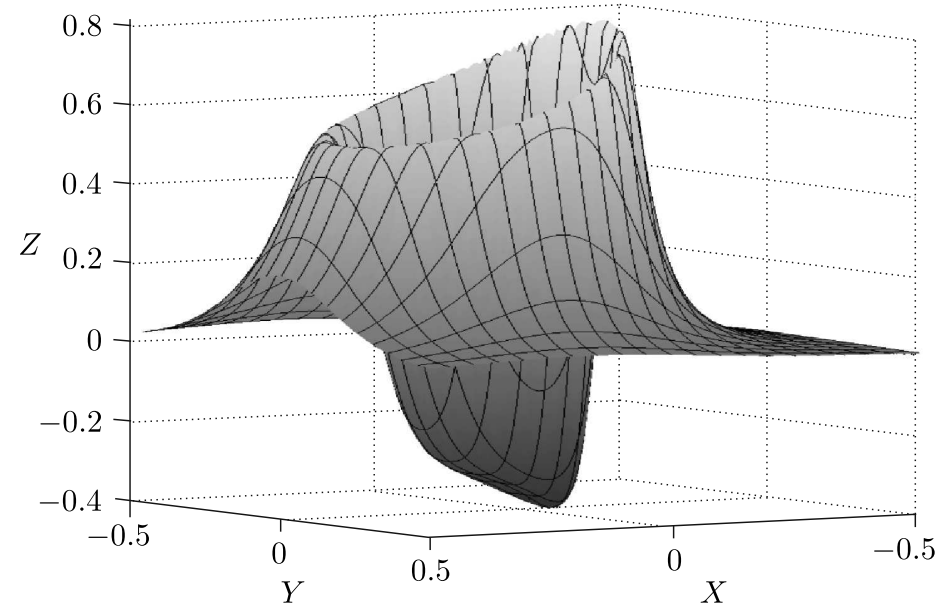

Рис. 1. Случай симметричного источника 


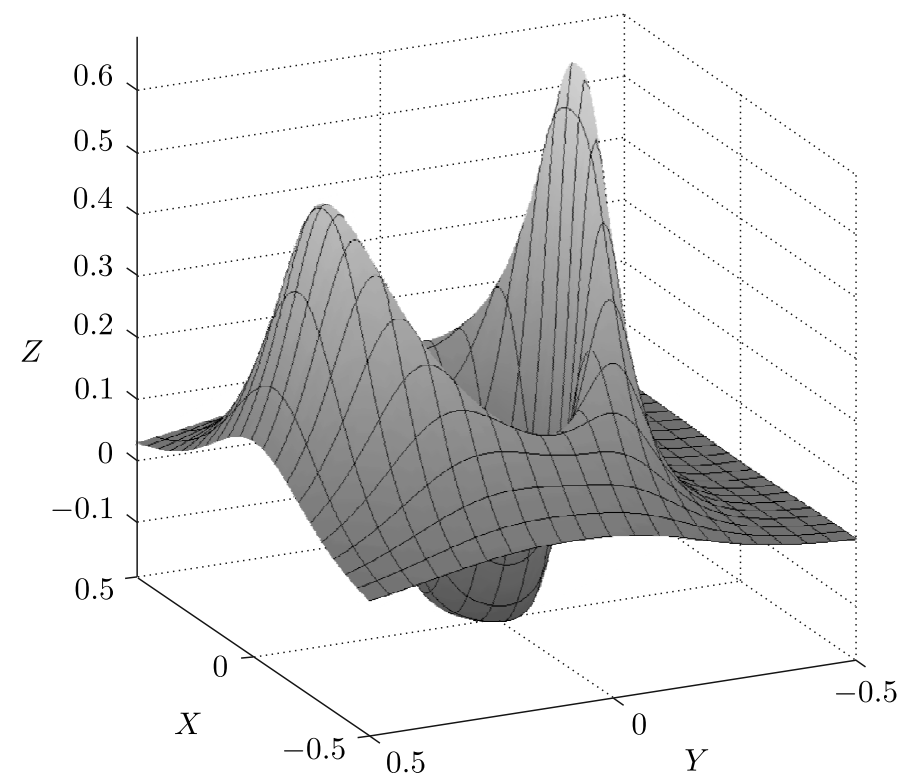

Рис. 2. Случай несимметричного источника. Угол $\theta=0$

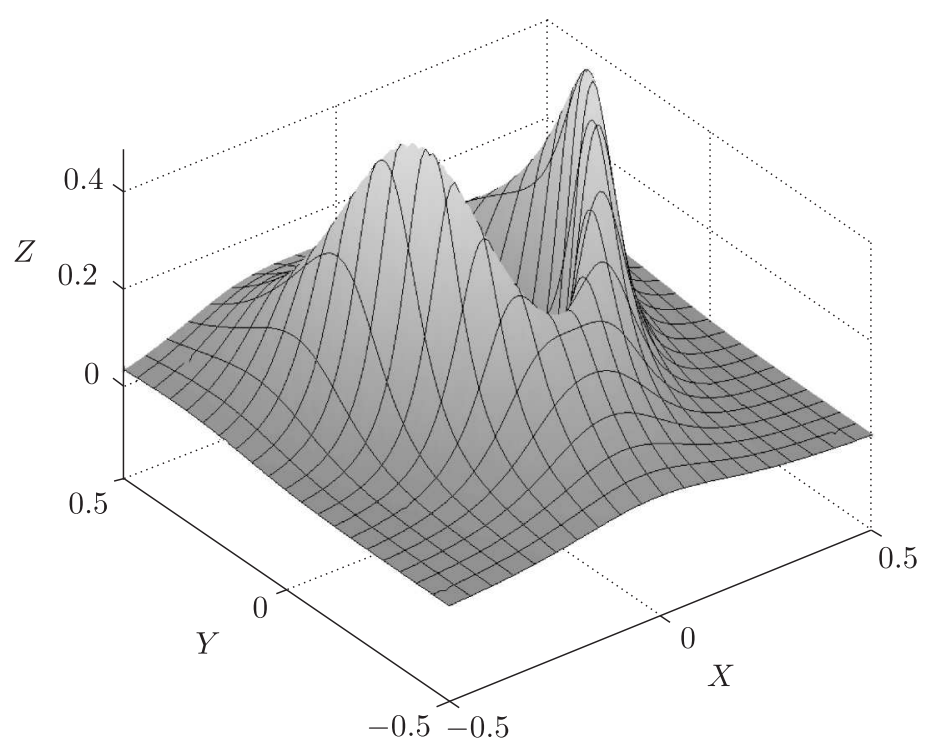

Рис. 3. Случай несимметричного источника. Угол $\theta=\pi / 2$

где $\theta=\psi-\psi_{1}, \cos \psi_{1}=x_{1} /|x|, \sin \psi_{1}=x_{2} /|x|$. Ответ для функции $\eta(x, t)$ получается следующий:

$$
\eta(x, t)=\sqrt{2 \pi} \cdot \operatorname{Re}\left\{\frac{a}{\left(a^{2}-1\right)^{3 / 2}}\right\},
$$

где $a=-t /|x|(C(0)-\langle\nabla C(0), x\rangle)+i \beta \mu /|x|$. 


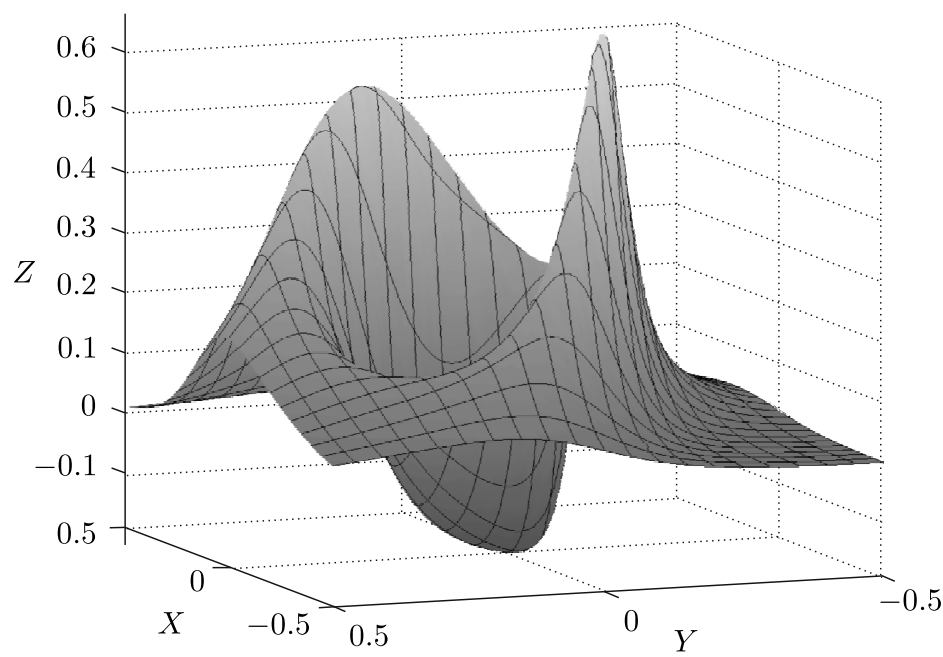

Рис. 4. Случай несимметричного источника. Угол $\theta=\pi / 4$

На рис. 1 изображен профиль решения $\eta(x, t)$ для симметричного источника в момент времени $t=0.2$. Здесь глубина в точке $(0,0)$ равна $1, \nabla C(0)=(2,0), \mu=0.08$. Из рисунка видно, что волна имеет разную высоту, причем, большей амплитуде соответствует меньшая глубина, а меньшей амплитуде - большая глубина.

Случай несимметричного источника. В том случае, когда источник является несимметричным, интеграл, который стоит в формуле (9), явно не вычисляется, и его надо считать численно. На рис. 2-4 изображены начальные стадии распространения волны цунами для разных углов $\theta$ в случае, когда глубина в точке $(0,0)$ равна $1, \nabla C(0)=(2,0), \mu=0.08$, $t=0.2$. На рис. 2 изображен случай несимметричного источника для $\theta=0$. Правому краю волны соответствует меньшая глубина, левому - большая. На рис. 3,4 изображены случаи несимметричного источника для $\theta=\pi / 2$ и $\theta=\pi / 4$ соответственно. На обоих рисунках меньшая глубина соответствует дальнему краю волны.

\section{СПИСОК ЦИТИРОВАННОЙ ЛИТЕРАТУРЫ}

[1] S. Yu. Dobrokhotov, A. I. Shafarevich, B. Tirozzi, Russ. J. Math. Phys., 15:2 (2008), 192-221. [2] S. Yu. Dobrokhotov, B. Tirozzi, C. A. Vargas, Russ. J. Math. Phys., 16:2 (2009), 228-245. [3] Е. Н. Пелиновский, Гидродинамика волн иунами, ИПФ РАН, Нижний Новгород, 1996. [4] С. Ю. Доброхотов, Б. Тироцци, А. И. Шафаревич, Матем. заметки, 82:5 (2007), 792-796. [5] С. Ю. Доброхотов, Б. И. Волков, С. Я. Секерж-Зенькович, Б. Тироцци, Фунамент. прикл. геофизика, 2 (2009), 15-29. [6] S. Wang, ASCE Specialty Conference on Coastal Hydrodynamics (June 29-July 1, 1987), Univ. of Delaware, 1987, 657-670.

\section{Д. А. Ложников}

Поступило

Институт проблем механики им. А. Ю. Ишлинского РАН,

18.11 .2010

Московский физико-технический институт

(государственный университет)

Исправленный вариант

E-mail: lozhnikov_d@list.ru

19.05.2011

\section{С. А. Сергеев}

Институт проблем механики им. А. Ю. Ишлинского РАН, Московский физико-технический институт

E-mail: sergeevse1@yandex.ru 